\title{
Comportements mécaniques : approches expérimentales et modélisations à différentes échelles
}

\author{
Renald Brenner ${ }^{1}$ et Éric Le Bourhis ${ }^{2}$
}
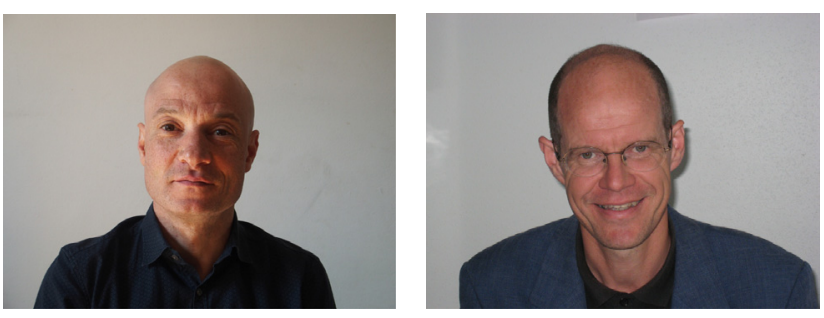

Le colloque « Comportements mécaniques » s'est déroulé du 24 au 28 novembre 2014 dans le cadre de la conférence Matériaux 2014 à Montpellier (http://www.sf2m.asso. fr/FFM_2014/mat2014/indexMat2014.html). Il a permis de réunir une communauté de chercheurs académiques et industriels, travaillant dans le domaine de la Mécanique des Matériaux, afin de discuter des dernières avancées et d'échanger sur les pratiques et les perspectives.

Ce colloque a été consacré aux études menées sur la description du comportement mécanique de matériaux très divers (polymères, verres, alliages métalliques, mousses, céramiques, géomatériaux, milieux granulaires, suspensions colloïdales, biomatériaux, etc.), en se fondant sur leur microstructure et/ou leurs mécanismes de déformation, d'endommagement et de rupture. Cette problématique inclut aussi bien l'élaboration (mise en forme, compaction, etc.) que la tenue en service normal et accidentel d'un matériau sous des chargements thermomécaniques variés. Les types de comportement abordés sont de natures très diverses : élastique fragile, viscoélastique, hyperélastique, élastoviscoplastique, endommageable.

Le colloque a accueilli plus de cent communications présentant des travaux expérimentaux, numériques et théoriques sur les relations «microstructure - mécanismes locaux - propriétés effectives ». Il s'est articulé autour de huit sessions thématiques portant sur : les approches probabilistes et l'homogénéisation, l'endommagement et la rupture, les sollicitations dynamiques, la rhéologie des milieux granulaires, la mécanique des polymères, la métallurgie mécanique, les interfaces dans les milieux solides et l'étude des comportements aux échelles micro et nanométriques.

Nous présentons dans ce numéro thématique, un ensemble d'articles issus d'une sélection de contributions au colloque, publiés après un processus d'évaluation et de révision. Ces articles offrent un aperçu de toutes les thématiques abordées au cours du colloque. L'ensemble du numéro regroupe des travaux fondamentaux et appliqués et permet de faire un point sur des sujets d'actualité dans le domaine de la Mécanique des Matériaux. Le lecteur y trouvera des travaux couvrant un large spectre de matériaux. Différentes modélisations des comportements élastique, fragile et ductile investiguées aux échelles pertinentes sont présentées et discutées.

Les éditeurs (R. Brenner et E. Le Bourhis) tiennent à remercier les organisateurs de Matériaux 2014 pour la programmation de ce colloque, les coordonnateurs des sessions thématiques (N. Bahlouli, R. Bargellini, N. Billon, R. Brenner, N. Carrère, O. Castelnau, J.P. Couzinie, J.Y. Delenne, B. Fayolle, L. Guillaumat, P. Ienny, J. Laverne, E. Le Bourhis, M. Lemaire, S. Mercier, Y. Monerie, D. Piot, F. Radjai, I. Raoult, M. Sauzay, H. Trumel, P. Viot, F. Willot) ainsi que les rapporteurs qui ont accepté d'expertiser les articles publiés dans ce numéro.

\footnotetext{
1 Éditeur Invité - « Matériaux \& Techniques », Institut d'Alembert, Sorbonne Universités, UPMC, UMR 7190 CNRS, 4 place Jussieu, CC 162, 75252 Paris Cedex 05, France

2 Éditeur - « Matériaux \& Techniques », Institut P', UPR 3346, CNRS - Université de Poitiers, Département de Physique et Mécanique des Matériaux, SP2MI - Boulevard Marie et Pierre Curie, BP 30179, 86962 Futuroscope Chasseneuil Cedex, France
} 